\title{
Comparing the role of subcision suction method with and without the injection of platelet-rich plasma in the treatment of depressed scars
}

\author{
Nouran Abd El-Aziz Abou Khedr ${ }^{1}$, Tarek Mahmoud Hussein ${ }^{1}$, Ayat Mohamed El-Sayed Abd \\ El-Fatah $^{2 *}$ \\ ${ }^{1}$ Department of Dermatology, Venereology and Andrology, Faculty of Medicine, Alexandria University, Egypt \\ ${ }^{2}$ Faculty of Medicine, Alexandria University, Egypt
}

\begin{abstract}
Among wound-healing modalities, platelet-rich plasma (PRP) has been used for wound healing, through the release of multiple growth factors. The platelets mediate wound healing by initiating the clotting pathway and the subsequent matrix remodelisation. The aim of the work was to study the role of injection of platelet-rich plasma in the treatment of scars. This study was conducted on thirty patients who were divided into two groups (A and B). Each group included fifteen patients. The two groups were randomly distributed by using individual sealed envelopes. Group A was subjected to subcision of their acne scars using Nokor needle, followed by suction. Group B patients were subjected to the same treatment but followed by an injection of platelet-rich plasma once per month over three months. In Group A, 6 patients (40\%) showed moderate improvement, 5 patients (33.3\%) showed slight improvement and 4 patients $(26.7 \%)$ showed significant improvement. In Group B, 7 patients $(46.7 \%)$ showed moderate improvement, 5 patients $(33.3 \%)$ showed marked improvement and 3 patients $(20 \%)$ showed significant improvement. There was a statistically significant difference on the independent observer's after-treatment assessment between the groups $(P=0.014)$. In conclusion, subcision suction leads to a persistent improvement of acne scars in a short time, and the coupling with injection of PRP is the most effective in the treatment of depressed facial scars.

Keywords: Platelet-rich plasma; scars; subcision; suction

Citation: Abou Khedr NAE, Hussein TM, Abd El-Fatah AME. Comparing the role of subcision suction method with and without the injection of platelet-rich plasma in the treatment of depressed scars. J Surg Dermatol 2017; 2(2): 88-97; http://dx.doi.org/10.18282/jsd. v2.i2.90.
\end{abstract}

*Correspondence to: Ayat Mohamed Elsayed Abd El-Fatah, Faculty of Medicine, Alexandria University, Egypt, ayatabdelfatah2016@gmail.com

Received: $5^{\text {th }}$ October 2016; Accepted: $14^{\text {th }}$ December 2016; Published Online: $14^{\text {th }}$ February 2017

\section{Introduction}

The process of wound healing is a complicated process whereby the skin repairs itself after injury ${ }^{[1,2]}$. The wound healing stages are in the order of: (1) inflammation, (2) granulation tissue formation, and (3) matrix remodelling $^{[3]}$.

Scarring leads to a difference in the normal structure and function of the skin, which manifests as a depressed area and with an alteration of skin quality, colour, vascularity, nerve supply and chemical properties ${ }^{[4]}$.

Scars can be classified as listed ${ }^{[5]}$ :

1. Fine line scars

2. Stretched scars

3. Scar contractures

4. Raised skin scars

a) Hypertropic scars

Copyright (C) 2017 Abou Khedr NAE, et al. This is an Open Access article distributed under the terms of the Creative Commons Attribution-NonCommercial 4.0 International License (http://creativecommons.org/licenses/by-nc/4.0/), permitting all non-commercial use, distribution, and reproduction in any medium, provided the original work is properly cited. 
b) Keloidal scars

5. Atrophic scars

a) Ice-pick scars

b) Rolling scars

c) Box-car scars

6. Intermediate scars

Subcision is described by Orentreich and Orentreich in $1995^{[6]}$ to illustrate the surgical procedure of atrophic scars using a Nokor needle which is inserted in the scar. The mechanism of this surgery is to separate the fibrous tissues which hold the scar to the underlying tissue ${ }^{[6]}$. Re-depression of scars must occur in the first two weeks after the procedure. Therefore, it was hypothesized that the suctioning of old scars prevent the re-depression by induction the hemorrhage in dermal pocket ${ }^{[7,8]}$.

Clinical trials have shown that the application of platelet-rich plasma (PRP) therapy to a wound can activate the healing process ${ }^{[9,10]}$. PRP also reduces the percentage of sternal infection ${ }^{[11]}$. Several recent reports have demonstrated the benefits of using topical application of PRP for improved post-operative outcomes following median sternotomy ${ }^{[11,12]}$, and so has PRP been used in the treatment of chronic wounds. Hence, an excellent management of chronic wounds is obtained toward improving natural healing ${ }^{[13]}$.

\section{Materials and Methods}

This interventional prospective randomized study was done on 30 patients who attended the dermatology outpatient clinic at Alexandria Main University Hospital. The patients were suffering from different types of depressed scars, and were enrolled in the study after obtaining informed written consent. The patients were divided into two groups (A and B) with 15 patients in each group:

1. Group A was subjected to subcision of their acne scars using Nokor needle, followed by suction starting on the third day after subcision and continued every other day using an electric-powered suction device for a period of two weeks.

2. Group B patients were subjected to subcision- suctioning similarly but followed by injection of platelet-rich plasma after their last session of suction with frequency of once per month for three months. Inclusion and exclusion criteria for patients were as follows:

Inclusion criteria:

1. Demonstrable post-acne, chicken-pox, traumatic and surgical depressed scars which were diagnosed on a clinical basis.

2. Patients who had given an informed consent to participate in the study.

3 . Patients with reasonable and realistic outcome expectations about the procedure.
Exclusion criteria:

1. Pregnant or lactating females.

2. Patients who were on systemic isotretinoin therapy in the previous twelve months.

3. Patients with susceptibility to keloid formation.

4. Patients taking drugs that prolong bleeding such as Aspirin and vitamin E.

5. Patients with active cystic acne lesions.

6. Patients with platelets dysfunction syndromes, bleeding diathesis.

7. Skin cancers, warts, solar keratoses and any skin infection.

8. Patients with liver cirrhosis or nephritis.

Assessment of the results was achieved objectively and the patients were digitally photographed using the same camera, lighting settings and patient positioning, before treatment and post-treatment at one month interval for a duration of three months. At the end of the study, a grading system of improvement for the evaluation was standardized. The severity of acne scarring was graded according to Goodman's qualitative global scarring grading system ${ }^{[14]}$ before and after treatment.

Subcision:

Anaesthesia: Topical anaesthesia (EMLA cream)

Patients' position: Semi-sitting position

Type of needle: Nokor needles (16-18 gauge)

Technique: Nokor needle was inserted into the scar with the bevel upwards parallel to the skin surface, and was moved back and forth in a fan-like motion under the scar. A snapping sound would be heard as the fibrous tissues separated. PRP was prepared by collecting the patient's own blood of about $20 \mathrm{cc}$ aliquots, placed sterilely into four tubes (each tube containing $50 \mathrm{~mL}$ sodium citrate anticoagulant). After centrifugation, the platelets and other vital growth proteins would be ready at the top of supernatant. The PRP was then drawn off, and the addition of calcium gluconate activated the PRP and resulted in the prompt release of $70 \%$ of growth factors from the $\alpha$-granules within 10 minutes (and nearly all the contents within an hour).We used a quartile grading scale ${ }^{[15]}$ to evaluate the effectiveness of the therapies, as stated below:

Slight improvement 0-25\%

Moderate improvement 25\%-49\%

Significant improvement 50\%-74\%

Marked improvement $\geqslant 75 \%$

\section{Ethics statement}

This study was approved by Ethical Committee of Faculty of Medicine, Alexandria University. 


\section{Results}

In Group A, the patients' ages ranged from 23-34 years with a mean value of $29.33 \pm 3.18$ years and the median value was 30; while in Group B, the ages ranged from 21-36 years with a mean age of $29.0 \pm 4.83$ years and the median age was 31 years. Out of 15 patients in Group A, 4 patients were males (26.7\%) and 11 patients were females (73.3\%). Group B consisted of 1 male (6.7\%) and 14 females (93.3\%). The groups were matched by age, gender and skin type. With regard to the distribution of scar based on etiology, the majority of patients in Group A (11 patients; 73.3\%) had acne scars, 2 patients (13.3\%) with chicken pox scars, 1 patient $(6.7 \%)$ with traumatic scars and another patient (6.7\%) had surgical scars. Group B included 13 patients (86.7\%) with acne scars, 1 patient (6.7\%) with chicken pox scars and 1 patient $(6.7 \%)$ with traumatic scars.

In Group A, 6 patients (40\%) showed moderate improvement, 5 patients $(33.3 \%)$ showed slight improvement and 4 patients (26.7\%) showed significant improvement. In Group B, 7 patients (46.7\%) showed moderate improvement, 5 patients $(33.3 \%)$ showed marked improvement, 3 patients (20\%) showed significant improvement. There was a statistically significant difference $(P=0.014)$ in an independent observer's after-treatment assessment between the groups according to a quartile grading scale ${ }^{[15]}$, which was used to evaluate the effectiveness of the therapies, as shown in Table $\mathbf{1}$ and Figure 1.

Table 1. Comparison between the two groups regarding independent observer's assessment at the end of the treatment

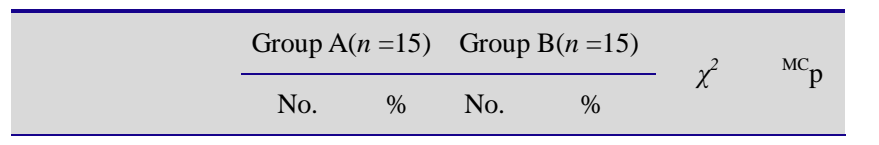

Independent ob-

server's assessment

at the end of the

treatment:

\begin{tabular}{|c|c|c|c|c|c|}
\hline Slight & 5 & 33.3 & 0 & 0.0 & \multirow{4}{*}{$10.030^{*} 0.014^{*}$} \\
\hline Moderate & 6 & 40.0 & 7 & 46.7 & \\
\hline Significant & 4 & 26.7 & 3 & 20.0 & \\
\hline Marked & 0 & 0.0 & 5 & 33.3 & \\
\hline \multicolumn{6}{|c|}{$\chi^{2}:$ Chi-square test } \\
\hline \multicolumn{6}{|c|}{$\begin{array}{l}\text { MC: Monte Carlo for chi-square test for comparing between } \\
\text { groups } \mathrm{A} \text { and } \mathrm{B}\end{array}$} \\
\hline \multicolumn{6}{|c|}{ *: Statistically significant at $P \leqslant 0.05$} \\
\hline \multicolumn{6}{|c|}{ Group $\mathrm{A}=$ without PRP } \\
\hline \multicolumn{6}{|c|}{ Group $B=$ with PRP } \\
\hline
\end{tabular}

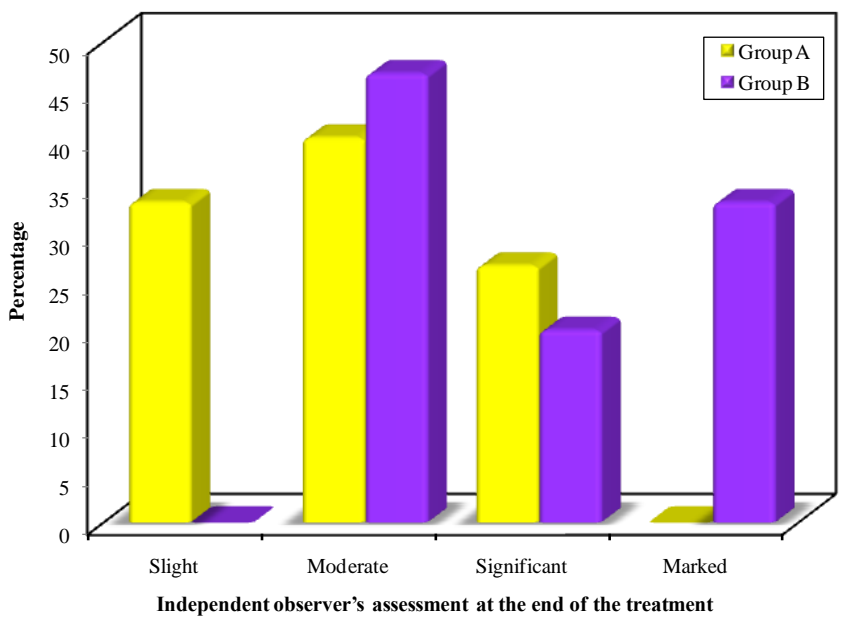

Figure 1. Comparison of improvement between the two groups regarding independent observer's assessment at the end of the treatment

Besides that, in Group A, 6 patients $(40 \%)$ perceived their improvement as slight, followed by 5 patients $(33.3 \%)$ who evaluated their improvement as moderate, 3 patients (20\%) as significant, and only 1 patient $(6.7 \%)$ as marked improvement.

In Group B, 7 patients (46.7\%) ranked their improvement as marked, 5 patients $(33.3 \%)$ as moderate improvement and 2 patients (13.3\%) as significant and only 1 patient $(6.7 \%)$ as slight improvement. There was a statistically significant difference $(P=0.038)$ in the patients' assessment score at the end of the treatment between the groups, as shown in Table 2 and Figure 2.

Table 2. Comparison between patients' assessment score at the end of the treatment between the two groups

\begin{tabular}{ccccc}
\hline & \multicolumn{2}{l}{$\operatorname{Group} \mathrm{A}(n=15)$} & Group B $(n=15)$ \\
\cline { 2 - 4 } & $\chi^{2} \quad{ }^{\mathrm{MC}} \mathrm{p}$ \\
\hline
\end{tabular}

\section{Patient assessment}

score at the end of

the treatment:

$\begin{array}{lrrrrr}\text { Slight } & 6 & 40.0 & 1 & 6.7 & \\ \text { Moderate } & 5 & 33.3 & 5 & 33.3 & \\ \text { Significant } & 3 & 20.0 & 2 & 13.3 & \\ \text { Marked } & 1 & 6.7 & 7 & 46.7 & \end{array}$

$\chi^{2}$ : Chi-square test

MC: Monte Carlo for chi-square test for comparing between groups A and $\mathrm{B}$

*: Statistically significant at $P \leqslant 0.05$

Group A = without PRP

Group B = with PRP 


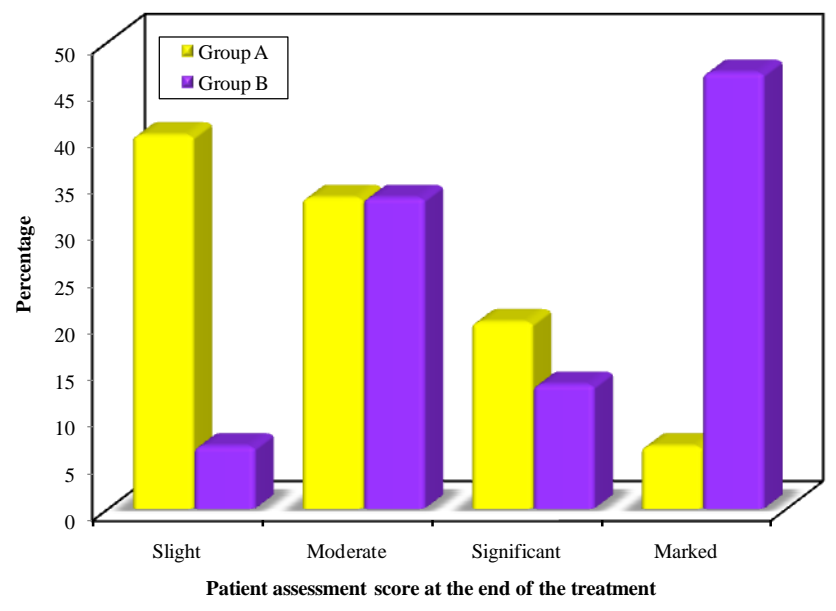

Figure 2. Comparison between patients' assessment scores at the end of the treatment between the two groups

According to pre-treatment grade of scars in Group A, the majority of patients (8 patients; $53.3 \%$ ) had moderate scars, followed by 4 patients (26.7\%) with mild scars and 3 patients (20\%) had severe scars. In the post-treatment grading of scars, 9 patients $(60 \%)$ had moderate scars, followed by 6 patients $(40 \%)$ with mild scars. There was a statistically significant difference $(P=0.025)$ regarding the grade of scars (pre-treatment and post-treatment) in Group A, as illustrated in Table 3 and Figure 3.

In pre-treatment grading of scars, the majority of patients in Group B (9 patients; 60\%) had moderate scars, followed by 3 patients (20\%) with mild scars and 3 patients $(20 \%)$ with severe scars. In post-treatment grading of scars, 5 patients (33.3\%) had moderate scars, followed by 9 patients $(60 \%)$ with mild scars and 1 patient $(6.7 \%)$ with severe scars. There was a statistically significant difference $(P=0.005)$ regarding the grade of scars (pre-treatment and post-treatment) in Group B, as shown in Table 3 and Figure 3.

The improvements are depicted in clinical images shown in Figures 4-10.

\section{Discussion}

The possible mechanism of PRP in the reconstruction of a depressed scar is by promoting the recovery of damaged skin through the numerous growth factors released, especially the platelet-derived growth factor. This growth factor may help to stimulate the production of other growth factors important in tissue remodelling, which promote connective tissue healing by up-regulating collagen and protein synthesis ${ }^{[16]}$. It has been suggested that autologous platelet-derived growth factors could be used in the treatment of depressed facial scars, which has been shown to accelerate tissue repair ${ }^{[16]}$.

Alam et al. evaluated the efficacy of subcision for the treatment of rolling acne scars in 40 patients. He demonstrated an approximately 50\% improvement of depressed
Table 3. Comparison between the studied groups according to grade of scars

\begin{tabular}{|c|c|c|c|c|c|}
\hline \multirow{2}{*}{ Grade of scars } & \multicolumn{2}{|c|}{ Pre-treatment } & \multicolumn{2}{|c|}{ Post-treatment } & \multirow{2}{*}{${ }^{\mathrm{MH}} \mathbf{p}_{\mathbf{1}}$} \\
\hline & No. & $\%$ & No. & $\%$ & \\
\hline \multicolumn{6}{|l|}{ Group A $(n=15)$} \\
\hline Mild & 4 & 26.7 & 6 & 40.0 & \\
\hline Moderate & 8 & 53.3 & 9 & 60.0 & $0.025^{*}$ \\
\hline Sever & 3 & 20.0 & 0 & 0.0 & \\
\hline \multicolumn{6}{|l|}{ Group B $(n=15)$} \\
\hline Mild & 3 & 20.0 & 9 & 60.0 & \\
\hline Moderate & 9 & 60.0 & 5 & 33.3 & $0.005^{*}$ \\
\hline Sever & 3 & 20.0 & 1 & 6.7 & \\
\hline$\chi^{2}$ & \multicolumn{2}{|c|}{0.347} & \multicolumn{2}{|c|}{2.630} & \\
\hline${ }^{\mathrm{MC}} \mathbf{p}$ & \multicolumn{2}{|c|}{1.000} & \multicolumn{2}{|c|}{0.275} & \\
\hline
\end{tabular}

$\chi^{2}$ : Chi-square test

MC: Monte Carlo for chi-square test for comparing between groups A and $\mathrm{B}$

${ }^{\mathrm{MH}} \mathrm{p}_{1}$ : $P$ values for marginal homogeneity test for comparing between pre- and post-treatment

*: Statistically significant at $P \leqslant 0.05$

Group A = without PRP

Group B = with PRP

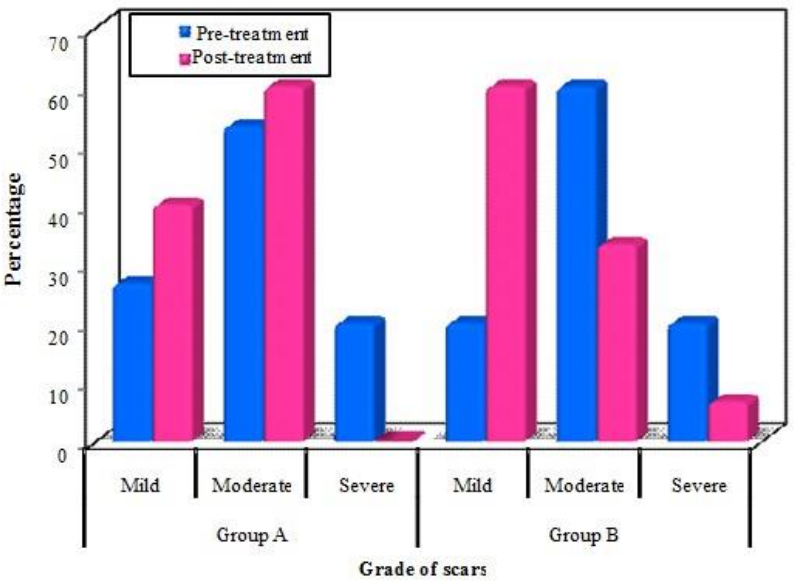

Figure 3. Comparison between the studied groups according to grade of scars (Qualitative scarring grading system) ${ }^{[14]}$

Mild: Mild atrophy or hypertrophic scars that may not be obvious at social distances of $50 \mathrm{~cm}$ or greater and may be covered adequately by makeup or the normal shadow of shaved beard hair in men or normal body hair if extrafacial.

Moderate: Moderate atrophic or hypertrophic scarring that may not be obvious at social distances of $50 \mathrm{~cm}$ or greater and is not covered easily by makeup or the normal shadow of shaved beard hair in men or body hair if extrafacial, but is still able to be flattened by manual stretching of the skin (if atrophic).

Severe: Severe atrophic or hypertrophic scarring that is evident at social distances of $50 \mathrm{~cm}$ or greater and is not covered easily by makeup or the normal shadow of shaved beard hair in men or body hair if extrafacial and is not able to be flattened by manual stretching of the skin. 


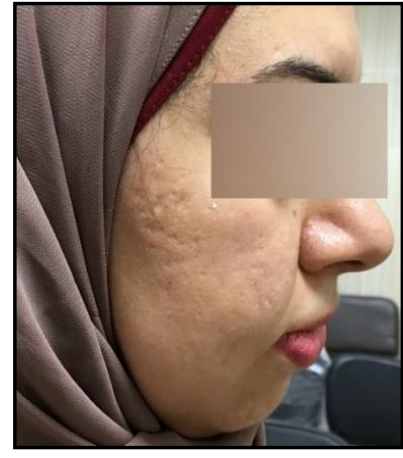

(A) Before

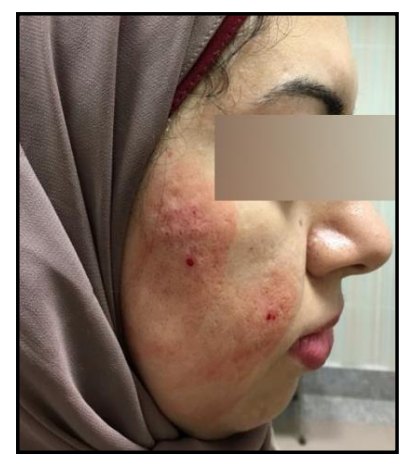

(B) Immediately after subcision

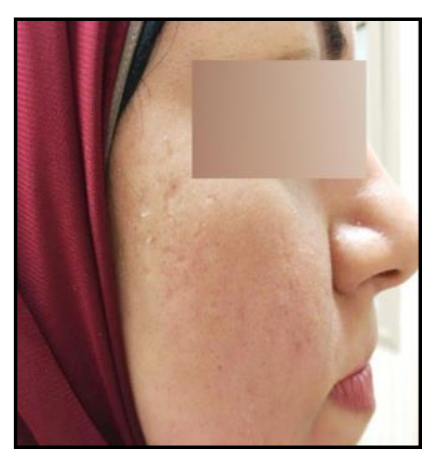

(C) Three months after PRP injection

Figure 4. Clinical image depicting 'Marked' response to PRP therapy

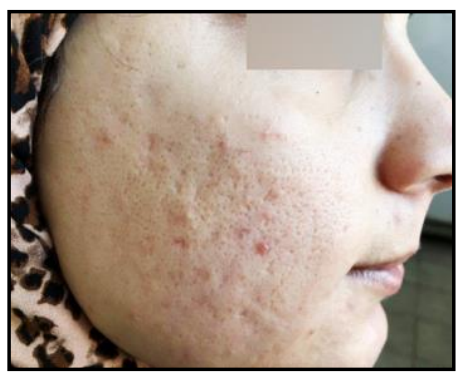

(A) Before

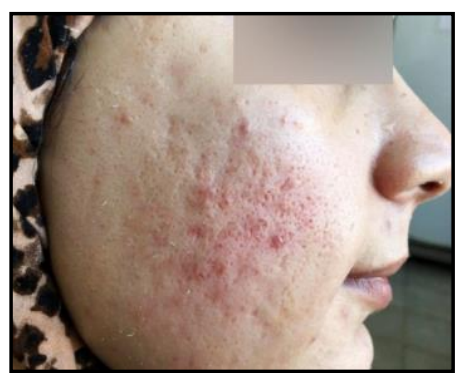

(B) Immediately after subcision

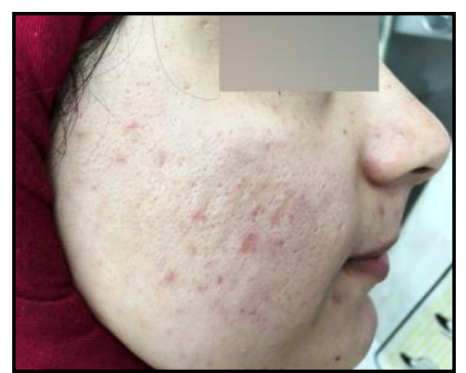

(C) Three months after PRP injection

Figure 5. A clinical image of acne scars showing 'Moderate' response following PRP treatment

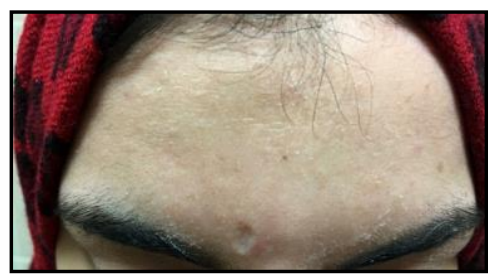

(A) Before

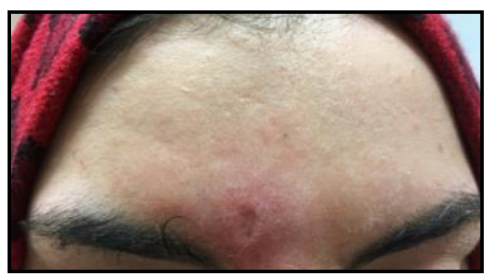

(B) Immediately after subcision

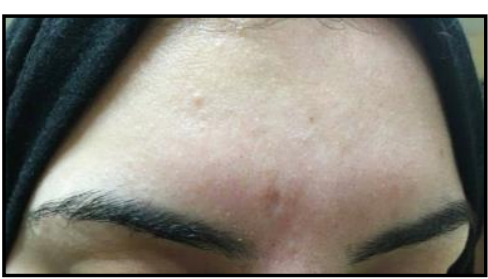

(C) Three months after PRP injection

Figure 6. Images showing 'Moderate' response of chicken-pox scar to PRP therapy

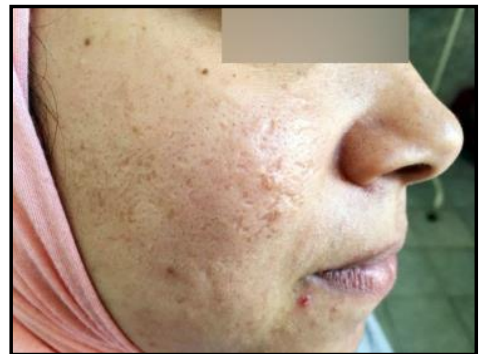

(A) Before

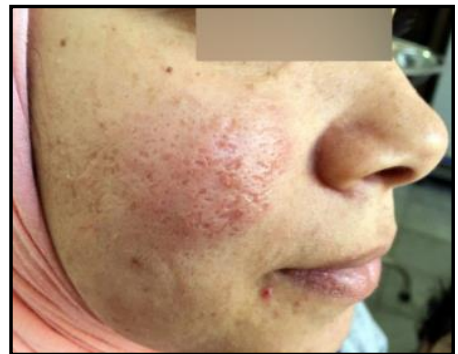

(B) Immediately after subcision

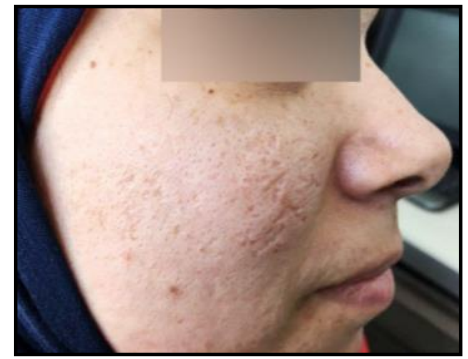

(C) Three months after subcision-suction

Figure 7. Clinical photograph depicting 'Slight' improvement after subcision-suction 


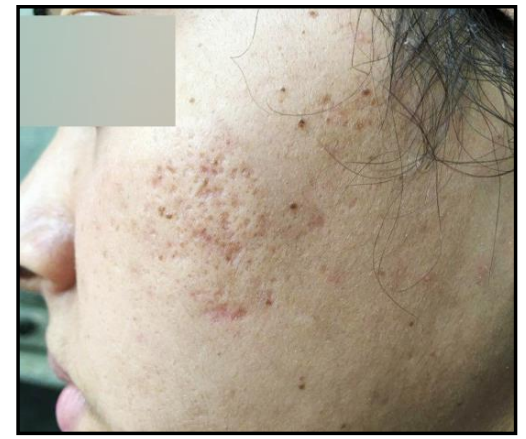

(A) Before

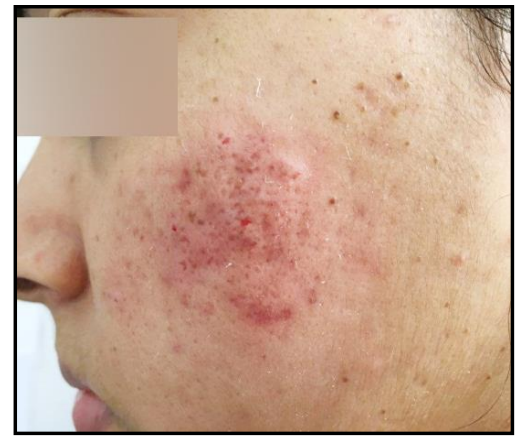

(B) Immediately after subcision

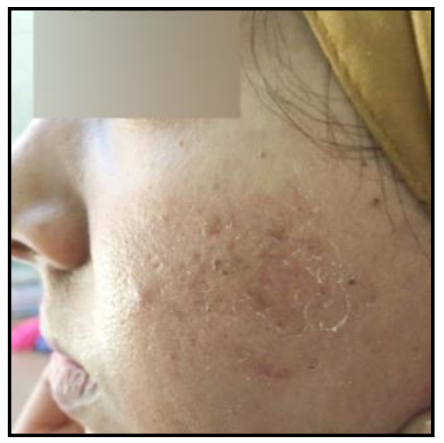

(C) Three months after PRP injection

Figure 8. Clinical photographs of acne scars showing 'Marked' improvement after PRP injection

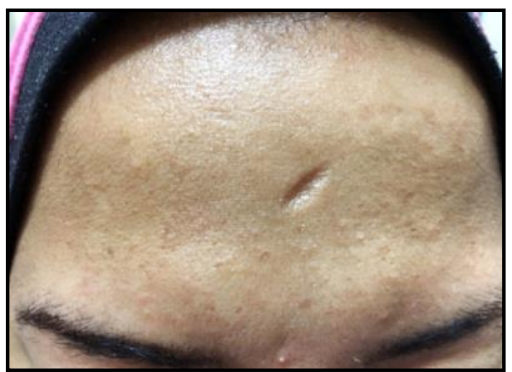

(A) Before

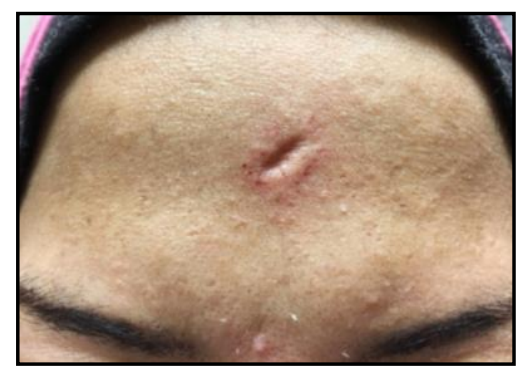

(B) Immediately after subcision

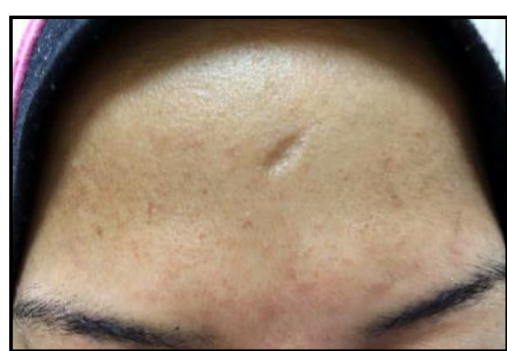

(C) Three months after subcision-suction

Figure 9. A photograph of post-traumatic scar presenting 'Slight' improvement after subcision-suction

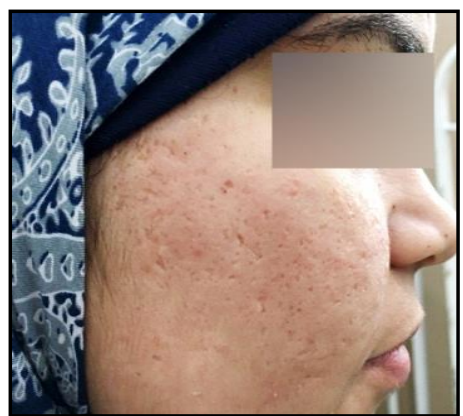

(A) Before

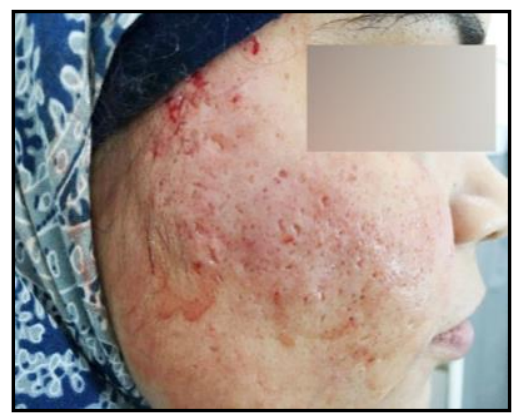

(B) Immediately after subcision

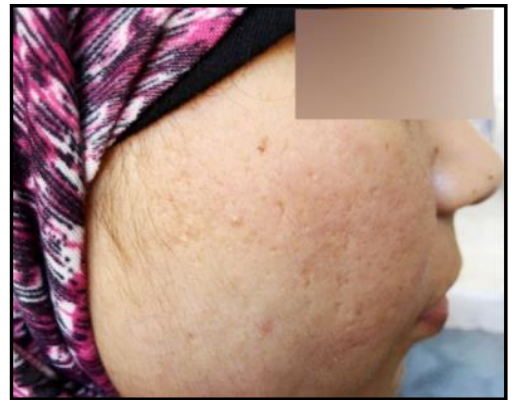

(C) Three months after PRP injection

Figure 10. Clinical images of acne scars, rolling and ice pick, showing 'Marked' improvement after PRP injection

scars, as observed in the present study. His evaluation was based on investigator's rating and patient's satisfaction, but the study lacked an objective scoring system and statistical analysis, in contrast to the current study ${ }^{[17]}$. Balighi et al. also used subcision for the treatment of depressed acne scars in 22 patients in absence of an objective scoring system ${ }^{[18]}$.

Goodman reported two cases with facial scars improved by subcision using a 19-gauge needle ${ }^{[19]}$. Fulchiero et al. reported a case with acne scars improved by subcision, followed by further improvement after nonablative resurfacing with $1320 \mathrm{~nm} \mathrm{Nd:YAG} \mathrm{laser}{ }^{[20]}$. These studies lacked patient quantity compared to our study. Photographic assessment in the current study fur- ther promoted that subcision is an effective treatment for acne scars.

Kang and colleagues have also reported significant improvement of atrophic acne scars after the use of triple combination therapy (CROSS technique, subcision and fractional laser), suggesting that combination therapy could be associated with better response than isolated modalities. This further substantiates the coupling of subcision and suctioning in the present study ${ }^{[21]}$.

In the adaptation of subcision, Goodman ${ }^{[22]}$ discussed the use of 19-gauge needles, while Hexsel and Mazzuco ${ }^{[23]}$ reported preferring 18-gauge Nokor needle. Later, it was found that better results could be achieved by using an 18 -gauge (1.5 inch) Nokor admix needle (Becton Dick- 
inson and Co., Franklin Lakes, $\mathrm{NJ})^{[24]}$ due to its triangular tip and the back-and-forth motion which allows for smooth and thorough separation of the fibrous cords. This explains the choice of Nokor needles in the current study.

Subjective and objective scorings were done by an independent dermatologist in order to minimize individual bias. Almost all patients treated with PRP stated that there was more reduction in the visibility of scars in comparison with the other group in the study. They also emphasized that the roughness of skin after treatment was higher in Group A than Group B.

The improvement rate in the current study following subcision-suction was clearly higher than that reported by Alam et al. ${ }^{[17]}$ and Balighi et al. ${ }^{[18]}$, who performed subcision as a solitary therapy for acne scars. They described an efficacy of 50\% with subcision alone. The enhanced efficacy by suction in the present study was maybe due to subcision alone, which releases the fibrotic tissue and thereby separating the underlying attachment and formation of blood dermal pocket beneath the scar. Blood not only acts as a short-term spacer to keep the tissue from early attachment, but also the subsequent organization of blood is thought to induce connective tissue formation and correction of the defect. The added benefit of repeated suction as a complementary treatment is most probably mediated by the suction causing repeated haemorrhage, delay in early attachment of dermal wound and more new connective tissue formation during the healing process of the subcised scar.

Subcision appears to be a simple, safe and well-tolerated surgical tool to improve acne scars. It is useful mainly for rolling depressed scars. It can be part of multiple types of treatments used for patients with acne scars. It can also lead to overall improvement in those unwilling to undergo other types of sophisticated treatments such as laser, dermabrasion or dermal fillers.

Redaelli et al. noticed improvement of acne scarring by PRP intradermal injection while using PRP for skin rejuvenation ${ }^{[25]}$. This led to the first recommendation for further trials to examine the benefit of PRP in acne scars. Lee et al. postulated that using PRP injection immediately after carbon dioxide laser resurfacing enhances the recovery of laser-damaged skin and synergistically improves the clinical appearance of acne scars ${ }^{[26]}$.

Therefore, PRP seems to be a promising new nonsurgical aesthetic modality. However, larger and well-controlled studies with prolonged follow-up periods should be performed to investigate its efficacy as a biostimulator and certify its long-term effects. The only trial that used the dermaroller combined with topical application of PRP was that of Fabbrocini et $a l^{[27]}$. They compared in a split-face study the effectiveness of skin needling alone and the combined use of skin needling plus topical PRP. Their results showed that the scar severity grade was greatly reduced on both sides of the face, but the improvement was more prominent on the side treated with both skin needling and PRP despite the inter-individual variation - $\mathrm{a}$ comparable finding which can be appreciated in present study.

In the present study, according to pre-treatment grade of scars in Group A, the majority of patients (53.3\%) had moderate scars, followed by $26.7 \%$ with mild scars and $20 \%$ had severe scars. In regards to post-treatment grade of scars, $60 \%$ had moderate scars, followed by $40 \%$ with mild scars. In Group B, the majority of patients $(60 \%)$ had moderate scars, followed by $20 \%$ with mild scars and $20 \%$ with severe scars in pre-treatment grade of scars. In regards to post-treatment grade of scars, 33.3\% of patients had moderate scars followed by $60 \%$ with mild scars and $6.7 \%$ with severe scars.

Also in the current study, according to the independent observer's rating improvement, the majority of patients (40\%) in Group A showed moderate improvement, $33.3 \%$ showed slight improvement, and $26.7 \%$ showed significant improvement. In Group B, $46.7 \%$ of patients showed moderate improvement, $33.3 \%$ showed marked improvement, and (20\%) showed significant improvement. This was in accordance with Hanradi et al. where $28.6 \%$ of their patients showed improvement $\geqslant 80 \%{ }^{[28]}$. This may be attributable to the inclusion of other types of scars as chicken pox scars, surgical and traumatic scars in the study. According to patients' assessment score at the end of the treatment between the two groups, the greater number of patients (40\%) in Group A perceived their improvement as slight, followed by $33.3 \%$ who evaluated their improvement as moderate, $20 \%$ as significant, and $6.7 \%$ as marked improvement. In Group B, $46.7 \%$ of patients ranked their improvement as marked, $33.3 \%$ as moderate improvement, $13.3 \%$ as significant, and $6.7 \%$ as slight improvement.

Nofal et al. treated 45 patients with atrophic acne scars with either PRP, $100 \%$ TCA CROSS, or skin needling with $\mathrm{PRP}^{[29]}$. An excellent-to-very-good rating was found in $46.7 \%$ of the PRP group, $26.7 \%$ of the TCA CROSS group, and $60 \%$ of the PRP with microneedling group. Similarly, Chawala completed microneedling upon 30 patients with facial acne and compared a split-face study of PRP and topical vitamin $\mathrm{C}^{[30]}$. The combination of microneedling with PRP had an excellent result in $18.5 \%$ of patients and was best with treating boxcar and rolling acne scars. Microneedling with topical vitamin C had a 7\% excellent improvement rate and showed more of an improvement in firmness and decreased post-inflammatory hyperpigmentation. 
Also, skin tightening to decrease the appearance of acne scars can be achieved through several methods. Garg and Baveja examined 50 patients with mildto-severe acne scarring and found that the combination of subcision, microneedling and 15\% TCA peel greatly improved both severe and mild acne scarring with a high level of patient satisfaction ${ }^{[31]}$.

Asif et al. treated 50 patients, all between 17-32 years of age with atrophic acne scars, with microneedling performed on both halves of the face. Intradermal injections as well as topical application of PRP were given on the right half of the face, while the left half of the face was treated with intradermal administration of distilled water with three treatment sessions given at intervals of one month ${ }^{[32]}$. Goodman's quantitative scale and qualitative scale were utilised for the final evaluation of results. The results of the right and the left halves showed $62.20 \%$ and $45.84 \%$ improvement, respectively, based on Goodman's quantitative scale.

Gawdat et al. conducted a study using PRP and Fractional $\mathrm{CO}_{2}$ Laser (FCL) and experienced that combined PRP and FCL-treated areas had a significantly better response $(P=0.03)$, fewer side effects and shorter downtime $(P=0.02)$ than FCL treated areas ${ }^{[33]}$.

Zhu et al. evaluated the efficacy of PRP and observed the overall degree of clinical improvement in the treatment of acne scars. Erbium FCL therapy was administered to patients with facial acne scars, and topical PRP gel was applied after laser therapy. In total, $68 \%$ and $91 \%$ of patients demonstrated a $50 \%$ improvement or greater of their scars on a quartile scale after the first and third treatment, respectively ${ }^{[34]}$. This study was not a split-face study, making it more difficult to assess how much of the improvement could be attributed to the PRP or the erbium FCL.

Another study utilized a split-face design to examine the treatment of patients with PRP injections on one half and saline injections on the other half of the face, after ablative carbon dioxide FCL therapy for the treatment of acne scars ${ }^{[26]}$. PRP treatment reduced the overall duration of erythema from $10.4 \pm 2.7$ to $8.6 \pm 2.0$ days. Furthermore, erythema was significantly less by day 4 as measured by a chromometer and the duration of edema was reduced by approximately one day on the PRP-treated side. The PRP-treated side showed notable improvements in the overall clinical appearance of acne scars compared to the control group as evaluated by independent dermatologists using a quartile grading system with a mean improvement of $2.7 \pm 0.7$ for the PRP group and $2.3 \pm 0.5$ for the control group ${ }^{[26]}$.

Both the topical and intradermal PRP treated groups had shorter recovery times and demonstrated significant improvements in the clinical appearance of acne scars compared to the control group that received FCL therapy only. Optical coherence tomography measurements of acne scar depth revealed that the FCL-only treatment group showed less improvement compared to the topical and intradermal PRP treatment.

There were no differences between the topical and intradermal PRP treatment groups, but the intradermal is considered the most used ${ }^{[33]}$. The present study documents the efficacy of combining subcision with suction in the treatment of atrophic acne scars. Subcision-suction has the potential to be used as the first step for acne and other depressed scars management. As multi-step treatment is necessary for an optimal correction of acne scars, it may be recommended to continue the treatment with other techniques or to repeat the subcision-suction method for several months. Combining subcision with injection of PRP can improve and/or eliminate depressed, broad-based acne scars. We propose that platelet-rich plasma is efficacious in the management of depressed facial scars. It can be combined with subcision to enhance the final clinical outcomes in comparison with subcision alone.

\section{Conclusion}

In conclusion, subcision is suitable for the different types of scars, especially rolling acne scars and depressed scars. However, for patients who want an overall improvement of acne scars, subcision can be an important component of a multistep treatment plan. Subcision-suction leads to a persistent improvement of acne scars in a short time, without considerable complication. Further studies evaluating the effectiveness of other surgical techniques in the management of depressed acne scars are recommended. Subcision followed by injection of PRP is most effective in the treatment of post-acne scars and all depressed facial scars. PRP injection is a new and promising modality for the treatment of scars without the risk of hyperpigmentation or scarring.

\section{Acknowledgements}

A line of appreciation to Prof. Dr. Nouran Abd El-Aziz Abou Khedr, Professor of Dermatology, Venereology and Andrology, for his unforgettable support as well as his generous efforts in the evaluation of this work. It is an honour to be supervised by him. We are also grateful to Prof. Dr. Tarek Mahmoud Hussein, Professor of Dermatology, who suggested this topic and also for his fruitful suggestions along with insistence in providing invaluable supervision of this study. 


\section{References}

1. Orgill DP, Blanco C. The pathophysiologic basis for wound healing and cutaneous regeneration. In: Biomaterials for treating skin loss. Cambridge: Elsevier, 2009. p 25. doi: 10.1201/9781420099904.ch4.

2. Rieger S, Zhao H, Martin P, Abe K, Lisse TS. The role of nuclear hormone receptors in cutaneous wound repair. Cell Biochem Funct 2015; 33: 1-13. doi: 10.1002/cbf.3086.

3. Stadelmann WK, Digenis AG, Tobin GR. Physiology and healing dynamics of chronic cutaneous wounds. Am J Surg 1998; 176(2A Suppl): 26S-38S.

4. Breathnach SM, McGrath JA. Wound healing. 7th ed. In: Burns T, Breathnach S, Cox N, Griffiths C (editors). Rook`s textbook of dermatology. Oxford: Blackwell Science Ltd, 2004. p. 11.1-11.25.

5. Bayat A, McGrouther DA, Ferguson MW. Skin scarring. Br Med J 2003; 326: 88-92. doi: 10.1136/bmj. 326.7380.88.

6. Orentreich DS, Orentreich N. Subcutaneous incisionless (subcision) surgery for the correction of depressed scars and wrinkles. Dermatol Surg 1995; 21: 543-549. doi: 10.1111/j.1524-4725.1995.tb00259.x.

7. Ghatan HEY. Morphologic eruptions. Dermatological differential diagnosis and pearls, 1st ed. New York: The Parthenon Publishing Group, 1994. pp 37-45.

8. Balighi K, Jamshidi S, Daneshpajooh M, Lajevardi V, Harandi SA, et al. Subcision for acne scarring with or without suctioning: A clinical trial. Iran J Dermatol 2011; 14: 95-99.

9. Driver VR, Hanft J, Fylling CP, Beriou JM, AutoloGel Diabetic Foot Ulcer Group. A prospective, randomized, controlled trial of autologous platelet-rich plasma gel for the treatment of diabetic foot ulcers. Ostomy Wound Manage 2006; 52(6): 68-87.

10. Frykberg RG, Driver VR, Carman D, Lucero B, Borris-Hale $\mathrm{C}$, et al. Chronic wounds treated with a physiologically relevant concentration of platelet-rich plasma gel: A prospective case series. Ostomy Wound Manage 2010; 56: 36-44.

11. Trowbridge CC, Stammers AH, Woods E, Yen BR, Klayman M, et al. Use of platelet gel and its effects on infection in cardiac surgery. J Extra Corpor Technol 2005; 37: 381-386.

12. Englert SJ, Estep TH, Ellis-Stoll CC. Autologous platelet gel applications during cardiovascular surgery: Effect on wound healing. J Extra Corpor Technol 2005; 37: 148-152.

13. Eisenbud D, Huang NF, Luke S, Silberklang M. Skin substitutes and wound healing: Current status and challenges. Wounds 2004; 16(1): 2-17.

14. Goodman GJ, Barton JA. Postacne scarring: A qualitative global scarring grading system. Dermatol Surg 2006; 32 : 1458-1466. doi: 10.1111/j.1524-4725.2006.32354.x.

15. Leheta T, El Tawdy A, Abdel Hay R, Farid S. Percutaneous collagen induction versus full-concentration trichloroacetic acid in the treatment of atrophic acne scars. Dermatol Surg 2011; 37: 207-216. doi: 10.1111/j.15244725.2010.01854.x.

16. Ganio C, Tenewitz FE, Wilson RC, Moyles BG. The treatment of chronic nonhealing wounds using autologous platelet-derived growth factors. J Foot Ankle Surg 1993; 32(3): 263-268.

17. Alam M, Omura N, Kaminer MS. Subcision for acne scarring: Technique and outcomes in 40 patients. Dermatol Surg 2005; 31: 310-317. doi: 10.1097/00042728200503000-00011.

18. Balighi K, Robati RM, Moslehi H, Robati AM. Subcision in acne scar with and without subdermal implant: A clinical trial. J Eur Acad Dermatol Venereol 2008; 22: 707-711. doi: 10.1111/j.1468-3083.2008.02583.x.

19. Goodman GJ. Therapeutic undermining of scars (Subcision). Australas J Dermatol 2001; 42: 114-117. doi: 10 . 1046/j.1440-0960.2001.00492.x.

20. Fulchiero GJ Jr, Parham-Vetter PC, Obagi S. Subcision and 1320-nm Nd:YAG nonablative laser resurfacing for the treatment of acne scars: A simultaneous split-face single patient trial. Dermatol Surg 2004; 30: 1356-1360. doi: 10.1111/j.1524-4725.2004.30411.x.

21. Kang W, Kim Y, Pyo W, Park SJ, Kim JH. Atrophic acne scar treatment using triple combination therapy: Dot peeling, subcision and fractional laser. Cosmet Laser Ther 2009; 11: 2-5. doi: 10.3109/14764170903134326.

22. Alsufyani MA, Alsufyani MA. Subcision: A further modification, an ever continuing process. Dermatol Res Pract 2012; 2012: 685347. doi:10.1155/2012/685347.

23. Ayeni O, Carey W, Muhn C. Acne scar treatment with subcision using a 20-G cataract blade. Dermatol Surg 2011; 37(6): 846-847. doi: 10.1111/j.1524-4725.2011.02010.x.

24. Jacob CI, Dover JS, Kaminer MS. Acne scarring: A classification system and review of treatment options. J Am Acad Dermatol 2001; 45: 109-117. doi: 10.1067/mjd. 2001.113451.

25. Redaelli A, Romano D, Marciano A. Face and neck revitalization with platelet-rich plasma (PRP): Clinical outcome in a series of 23 consecutively treated patients. J Drugs Dermatol 2010; 9: 466-472.

26. Lee J, Kim B, Kim M, Mun S. The efficacy of autologous platelet rich plasma combined with ablative carbon dioxide fractional resurfacing for acne scars: A simultaneous split-face trial. Dermatol Surg 2011; 37(7): 931-938. doi: 10.1111/j.1524-4725.2011.01999.x.

27. Fabbrocini G, De Vita V, Pastore F, Panariello L, Fardella $\mathrm{N}$, Sepulveres R. et al. Combined use of skin needling and platelet-rich plasma in acne scarring treatment. J Cosmet Dermatol 2011; 24: 177-183.

28. Harandi SA, Balighi K, Lajevardi V, Akbari E. Subcision-suction method: A new successful combination therapy in treatment of atrophic acne scars and other depressed scars. J Eur Acad Dermatol Venereol 2011; 25: 92-99. doi: 10.1111/j.1468-3083.2010.03711.x.

29. Nofal E, Helmy A, Nofal A, Alakad R, Nasr M. Platelet-rich plasma versus CROSS technique with $100 \%$ trichloroacetic acid versus combined skin needling and platelet rich plasma in the treatment of atrophic acne scars: A comparative study. Dermatol Surg 2014; 40(8): 864-783. doi: 10.1111/dsu.0000000000000091.

30. Chawla S. Split face comparative study of microneedling with PRP versus microneedling with vitamin $\mathrm{C}$ in treating atrophic post acne scars. J Cutan Aesthet Surg 2014; 7: 
209-212. doi: 10.4103/0974-2077.150742.

31. Garg S, Baveja S. Combination therapy in the management of atrophic acne scars. J Cutan Aesthet Surg 2014; 7: 18-23. doi: 10.4103/0974-2077.129964.

32. Asif M, Kanodia S, Singh K. Combined autologous platelet-rich plasma with microneedling verses [sic] microneedling with distilled water in the treatment of atrophic acne scars: A concurrent split-face study. J Cosmet Dermatol 2016; 1-10. doi: 10.1111/jocd.12207.
33. Gawdat HI, Hegazy RA, Fawzy MM, Fathy M. Autologous platelet rich plasma: Topical versus intradermal platelet rich plasma after fractional ablative carbon dioxide laser treatment of atrophic acne scars. Dermatol Surg 2014; 40: 152-161. doi: 10.1111/dsu.12392.

34. Zhu JT, Xuan M, Zhang YN, Liu HW, Cai JH, Wu YH, et al. The efficacy of autologous platelet-rich plasma combined with erbium fractional laser therapy for facial acne scars or acne. Mol Med Rep 2013; 8: 233-7. 